\title{
Classical blood biomarkers identify patients with higher risk for relapse 6 months after alcohol withdrawal treatment
}

\author{
Florian J. Raabe ${ }^{1,2}\left(\right.$ Elias Wagner $^{1} \cdot$ Judith Weiser $^{1} \cdot$ Sarah Brechtel $^{1} \cdot$ David Popovic $^{1,2} \cdot$ Kristina Adorjan $^{1,3}$. \\ Oliver Pogarell ${ }^{1} \cdot$ Eva Hoch $^{1,4} \cdot$ Gabriele Koller $^{1}$
}

Received: 14 April 2020 / Accepted: 16 June 2020 / Published online: 5 July 2020

(c) The Author(s) 2020

\begin{abstract}
This naturalistic study among patients with alcohol dependence examined whether routine blood biomarkers could help to identify patients with high risk for relapse after withdrawal treatment. In a longitudinal study with 6-month follow-up among 133 patients with alcohol dependence who received inpatient alcohol withdrawal treatment, we investigated the usefulness of routine blood biomarkers and clinical and sociodemographic factors for potential outcome prediction and risk stratification. Baseline routine blood biomarkers (gamma-glutamyl transferase [GGT], alanine aminotransferase [ALT/GPT], aspartate aminotransferase [AST/GOT], mean cell volume of erythrocytes [MCV]), and clinical and sociodemographic characteristics were recorded at admission. Standardized 6 months' follow-up assessed outcome variables continuous abstinence, days of continuous abstinence, daily alcohol consumption and current abstinence. The combined threshold criterion of an AST:ALT ratio $>1.00$ and $\mathrm{MCV}>90.0 \mathrm{fl}$ helped to identify high-risk patients. They had lower abstinence rates $(P=0.001)$, higher rates of daily alcohol consumption $(P<0.001)$ and shorter periods of continuous abstinence $(P=0.027)$ compared with lowrisk patients who did not meet the threshold criterion. Regression analysis confirmed our hypothesis that the combination criterion is an individual baseline variable that significantly predicted parts of the respective outcome variances. Routinely assessed indirect alcohol biomarkers help to identify patients with high risk for relapse after alcohol withdrawal treatment. Clinical decision algorithms to identify patients with high risk for relapse after alcohol withdrawal treatment could include classical blood biomarkers in addition to clinical and sociodemographic items.
\end{abstract}

Keywords Alcohol dependence $\cdot$ Alcohol use disorder $\cdot$ Withdrawal treatment $\cdot$ Risk for relapse $\cdot$ Outcome $\cdot$ Blood biomarkers

Communicated by Andrea Schmitt.

Florian J. Raabe, Elias Wagner, Eva Hoch and Gabriele Koller have contributed equally.

Florian J. Raabe

Florian.Raabe@med.uni-muenchen.de

1 Department of Psychiatry and Psychotherapy, University Hospital, LMU Munich, Nussbaumstrasse 7, 80336 Munich, Germany

2 International Max Planck Research School for Translational Psychiatry (IMPRS-TP), Kraepelinstrasse 2-10, 80804 Munich, Germany

3 Institute of Psychiatric Phenomics and Genomics (IPPG), University Hospital, LMU Munich, Nussbaumstrasse 7, 80336 Munich, Germany

4 Division of Clinical Psychology and Psychological Treatment, Department of Psychology, LMU Munich, Leopoldstrasse 13, 80802 Munich, Germany

\section{Introduction}

Alcohol use disorder (AUD) is a severe, chronic substance use disorder with a significant individual and socio-economic burden; it is highly prevalent and affects approx. 100 million people globally [12]. Furthermore, about 40-60\% of patients with AUD are presumed to relapse within the first year after treatment [11,27]. Due to limited resources of health care systems, the effective allocation of medical treatment to individual patients is a major challenge [36]. A promising strategy to improve the outcome might be to individualize treatment and to identify patients on the basis of applicable subgroups and endophenotypes [24].

Biological and non-biological variables (e.g. sociodemographic, environmental and clinical factors) have been previously associated with the risk of relapse in patients or subgroups of patients with AUD [1, 8, 13, 29, 32, 38]. 
Despite neuroimaging [8, 13], genetics [29] and the measurement of neurometabolites [29] being sophisticated methods to estimate resilience and the risk of relapse after treatment, routine blood biomarkers might be a more feasible and cost-effective means of assessing relapse risk. The classical indirect alcohol blood biomarkers are the liver enzymes $\gamma$-glutamyl transferase (GGT), alanine aminotransferase (ALT), aspartate aminotransferase (AST) and the mean cell volume of erythrocytes (MCV). All of them reflect chronic excessive drinking and are basic parameters for clinical laboratories in daily routine care settings [3,25]. A previous study investigated the ability of a combination of an AST:ALT ratio $>1.00$ and an MCV $>90.0$ fl to identify patients with alcohol dependence (AD) in a cohort of individuals with and without physical diseases and with and without elevated transaminase levels [17]. The combination of these routine blood biomarkers resulted in a remarkably high sensitivity of $97.3 \%$ and specificity of $88.9 \%$ [17].

So far, most studies have investigated the use of blood biomarkers (1) to identify patients with $\mathrm{AD}$ and/or (2) as a screening tool for potential alcohol consumption before sample collection and/or (3) to detect relapse and monitor abstinence in patients with $\mathrm{AD}$ [2, 3, 20, 42].

Blood biomarkers might not only help to identify patients with $\mathrm{AD}$ or help to monitor therapeutic strategies and abstinence but might also serve as prognostic markers to assess the risk of relapse and thus might support future clinical decision algorithms [31]. Indirect alcohol biomarkers represent an objective measurement for chronic cellular impairment that correlates with previous alcohol consumption [35] and, therefore, might reflect the severity of AD and consequently the risk of relapse after withdrawal treatment. In this context, in a sample of individuals with alcohol abuse and dependence, higher levels of the blood biomarker CDT and the liver function enzymes ALT and GGT were predictors for drunk-driving recidivism [23]. Furthermore, higher GGT scores were associated with unfavourable outcomes during and after pharmacotherapy for $\mathrm{AD}[15,16]$. In a longitudinal study assessing post-treatment outcomes in an outpatient sample with one-year follow-up after treatment, higher GGT, AST, ALT, MCV scores and AST/ALT ratios were associated with unfavourable outcomes with regard to drinking-behaviour [14].

In this study, we investigated the potential of biological (i.e. routine blood biomarkers, breath alcohol and alcoholic liver disease) and non-biological factors (i.e. sociodemographic and clinical variables) to estimate the risk for relapse after inpatient withdrawal treatment. Therefore, we conducted a naturalistic longitudinal study among patients with $\mathrm{AD}$ in a routine care setting with a 6-month followup to evaluate whether baseline both biological and nonbiological factors, assessed at admission to inpatient alcohol withdrawal treatment, are associated with long-term outcome after completion of treatment.

\section{Methods}

\section{Design}

Included patients received routine inpatient care with a standardized multimodal and multi-professional qualified (extended) withdrawal treatment (QWT) at the inpatient addiction unit at the Department of Psychiatry and Psychotherapy, University Hospital, LMU Munich, Munich, Germany. Information on biological and non-biological baseline variables at admission was obtained from standardized electronic medical records. Previous inpatients were contacted and interviewed 6 months after completion of treatment to assess relevant outcome variables. We chose 6 months as the follow-up period since this is presumed to be a good predictor for 5-year outcome [40]. The study was approved by the local ethics committee of the Faculty of Medicine, LMU Munich, Germany (project number 585-16).

\section{Participants}

The study was conducted between September 2016 and January 2018. Inclusion criteria were: (1) patients with a confirmed diagnosis of $\mathrm{AD}$ (F10.2) according to the International Classification of Diseases and Related Health Problems 10th Revision (ICD-10), (2) treated at the inpatient addiction unit between March 18, 2016 and July 26, 2017, (3) age $\geq 18$ years and (4) participation in QWT for $\geq 5$ days.

On the basis of electronic records, we identified 338 patients (71.3\% male, $28.7 \%$ female, mean [SD] age: 46.7 [12.6] years) with a recorded diagnosis of $\mathrm{AD}$ according to ICD-10 who had received $\geq 5$ days of QWT in the period of interest. To exclude patients with a diagnosis other than $\mathrm{AD}$ (e.g. multiple drug abuse [F19.2]), trained physicians reassessed each patient's diagnosis and excluded 24 patients with a diagnosis other than AD (e.g. multiple drug abuse [F19.2]). Thus, we contacted 314 former inpatients. 51.2\% (161 out of 314) of previous inpatients could not be reached and 1 person $(0.3 \%)$ declined to participate in the followup interview. Of the successfully contacted people, 19 were excluded due to a protocol violation (they were contacted earlier than 6 months after QWT). Thus, a total of 133 out of $314(42.4 \%)$ former inpatients ( $71.4 \%$ males, $28.6 \%$ females, mean [SD] age 47.4 [13.0] years) were contacted successfully 6 months after QWT and interviewed by telephone. Figure 1 shows a detailed overview of the selection process.

Only two baseline features significantly differed between the study participants who were successfully contacted after 6 months and those who were not reached by telephone 
Fig. 1 Overview of the selection process for the longitudinal study with 6-month follow-up among 133 patients with alcohol dependence who received inpatient alcohol withdrawal treatment

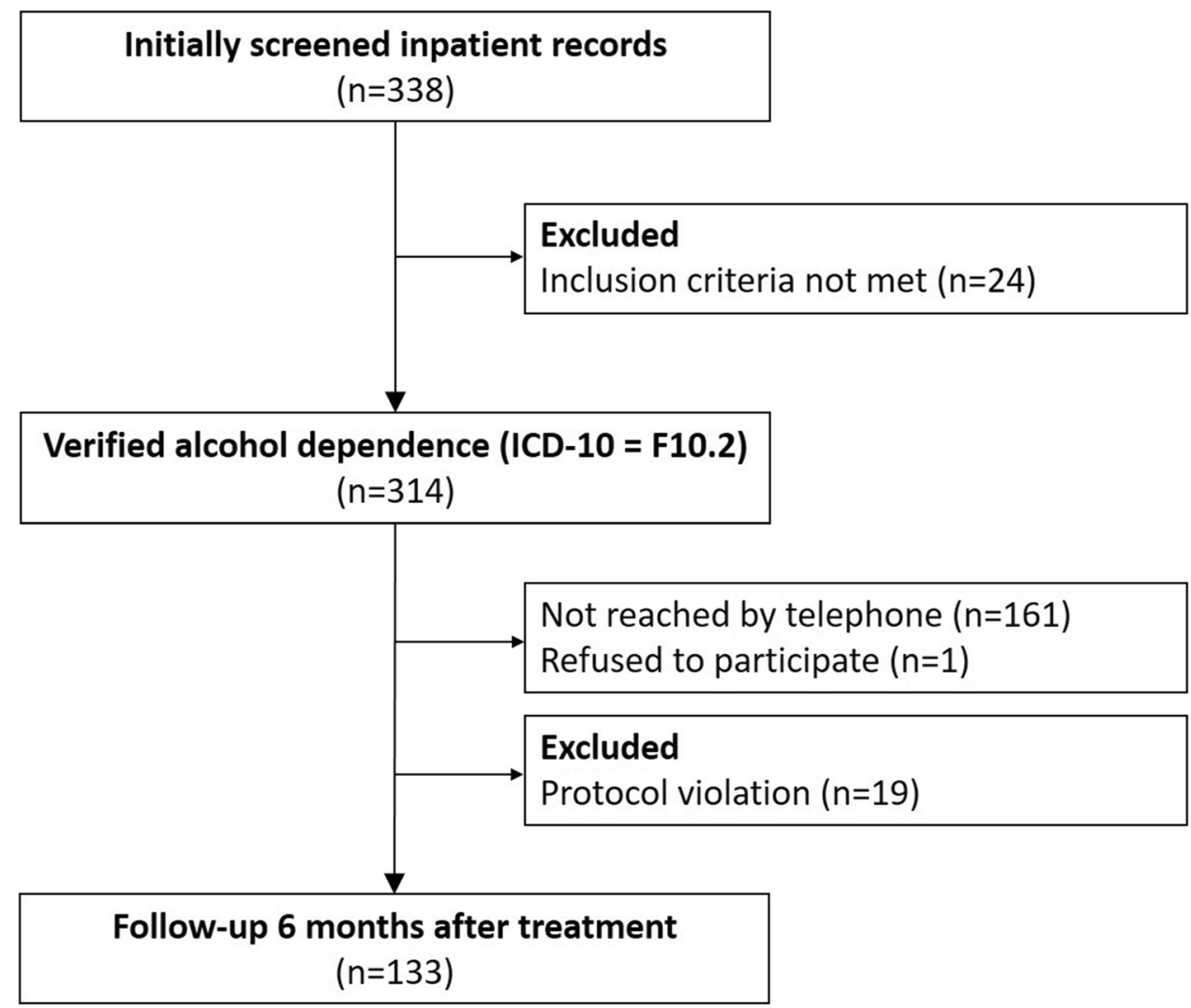

or were excluded (non-follow-up sample). More former inpatients in the non-follow-up group had an immigration background than in the participant group (43.1\% vs. $\left.26.1 \%, \chi^{2}=4.263, d f=1, P=0.039\right)$ and the prevalence of alcoholic liver disease detected by ultrasound was higher in the non-follow-up group compared to the participant group (63.1\% vs. $\left.49.2 \%, \chi^{2}=5.682, d f=1, P=0.017\right)$. All other baseline features including liver enzymes (AST, ALT, GGT) and MCV did not significantly differ between the follow-up group and the non-follow-up group.

\section{Setting}

QWT is a multimodal and multi-professional inpatient detoxification and treatment programme [5]. It is usually planned for 14-21 days and is a highly standardized part of routine clinical care in the inpatient addiction unit at the Department of Psychiatry and Psychotherapy, University Hospital, LMU Munich, Munich, Germany. Medical treatment to control acute alcohol withdrawal symptoms included the application of benzodiazepines (oxazepam) and alpha-2 noradrenergic agonists (clonidine). Patients received medication as needed, according to their clinical condition. Patients with a history of at least one epileptic seizure were additionally treated with a fixed dose of anticonvulsants (carbamazepine or levetiracetam). Patients also received their previously prescribed medication for their physical and psychiatric conditions. In addition to symptom-specific or prophylactic treatment of withdrawal symptoms, QWT includes psychotherapeutic interventions covering motivational enhancement, cognitive behavioural therapy and relapse prevention; occupational therapy; physiotherapy; work-related therapy, art and music therapy; and attendance at self-help groups (e.g. AA groups). Abstinence during QWT was monitored with breath analysers for alcohol.

\section{Baseline data}

Baseline data at admission to inpatient treatment were extracted from standardized electronic medical records. At admission, all patients were interviewed with a standard questionnaire based on the European Addiction Severity Index (EuropASI) [19], a semi-structured assessment instrument for drug and alcohol abuse. The clinical records of study participants during QWT were used to assess baseline data of the patients. We collected baseline data on both biological (routine blood biomarkers [GGT, AST, ALT, MCV], breath alcohol and alcoholic liver disease detected by ultrasound) and non-biological factors (sociodemographic and clinical variables; see Table 1 for details). We used the combined threshold criterion of 


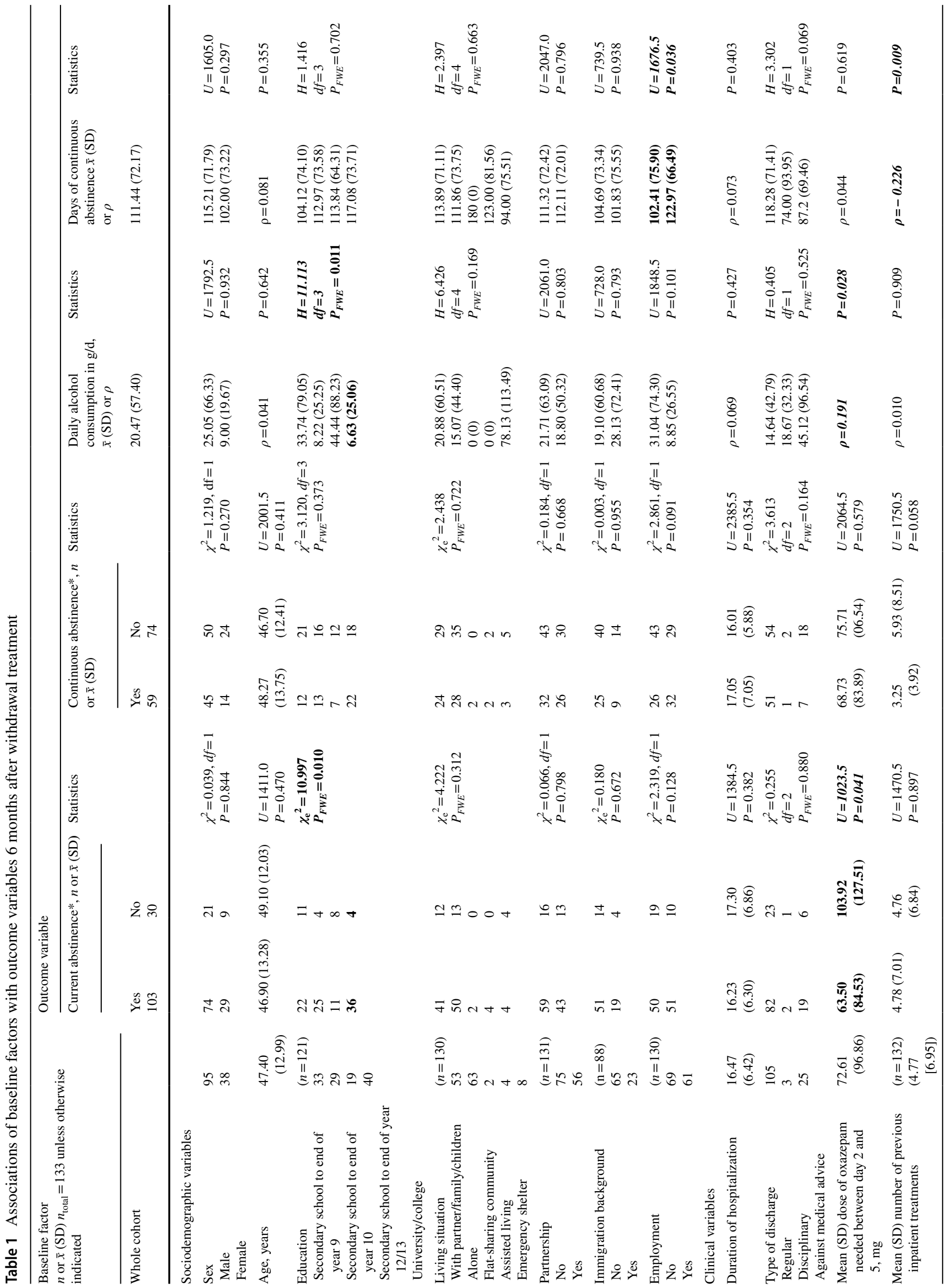




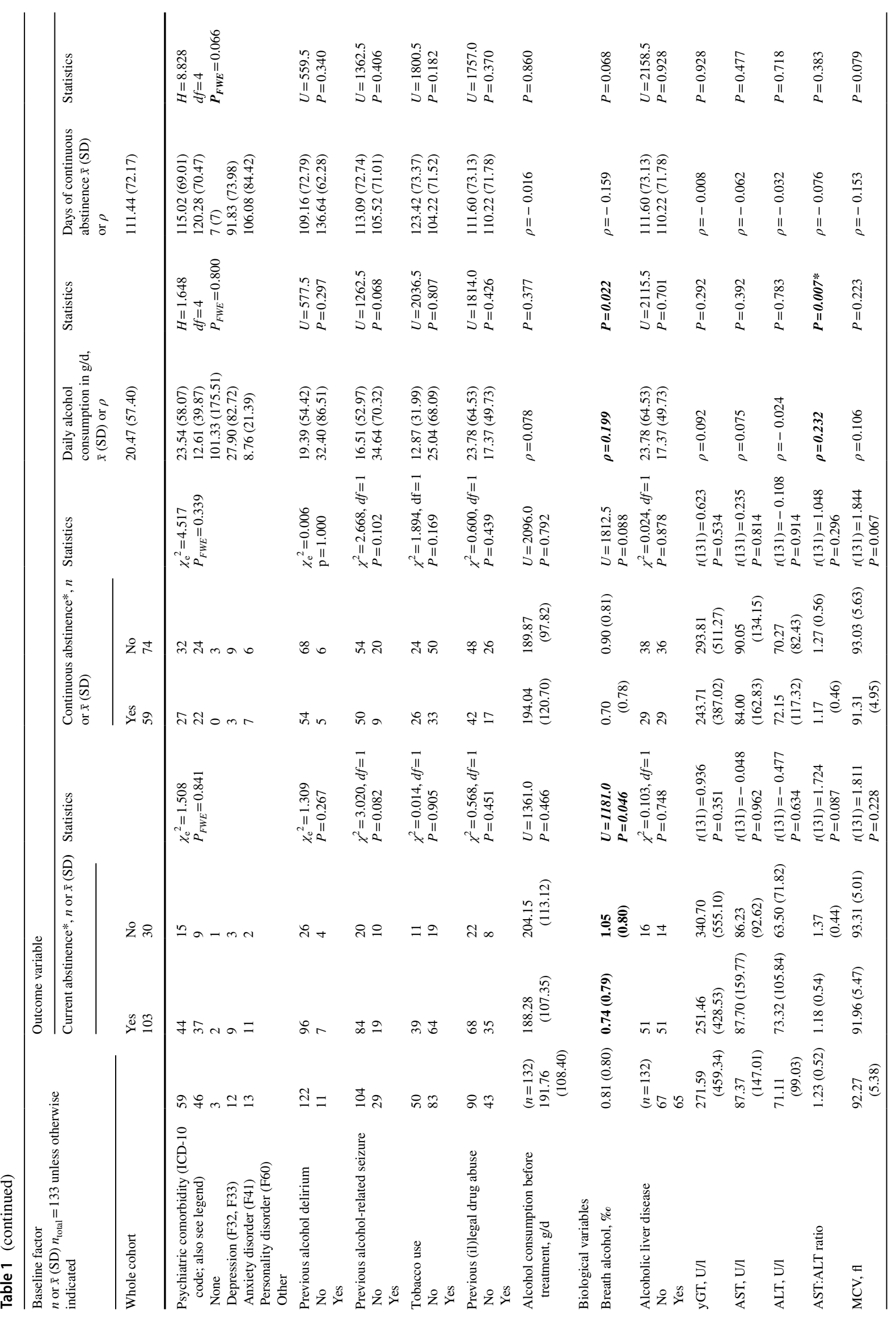




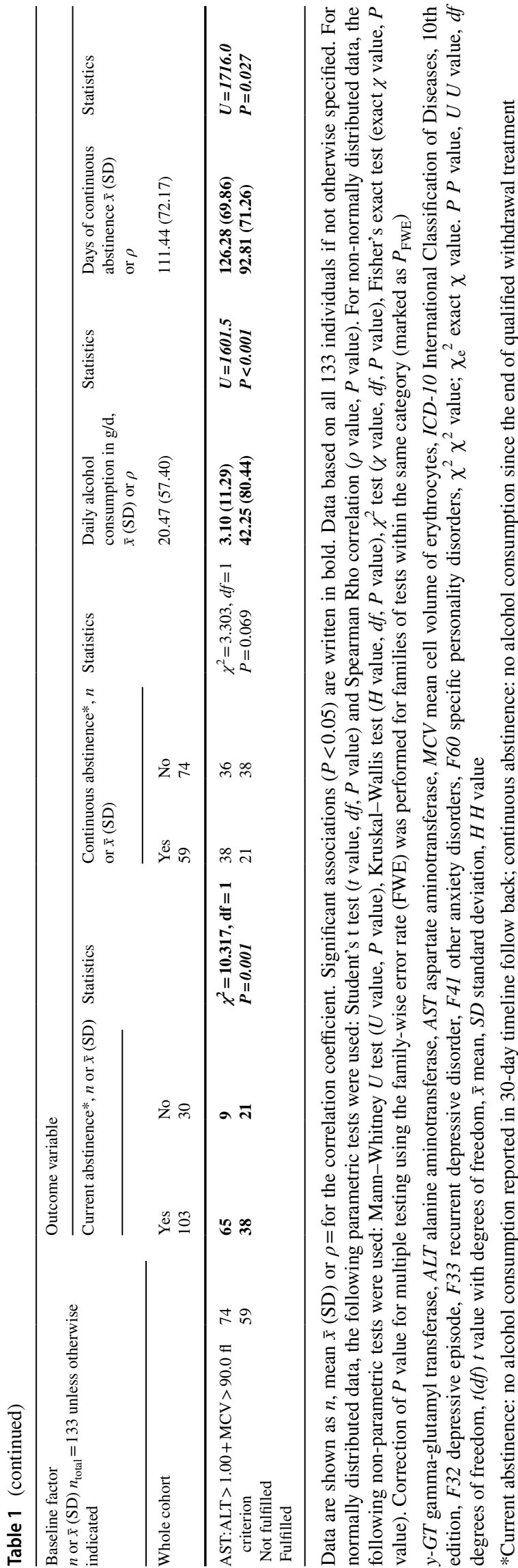

an AST:ALT > 1.00 and a MCV > $90.0 \mathrm{fl} \mathrm{[17]} \mathrm{to} \mathrm{divide}$ participants into "low-risk patients", who did not fulfil the criterion, and "high-risk patients", who did. Baseline blood samples were collected within $72 \mathrm{~h}$ after admission and an ultrasound scan was performed to detect alcoholic liver diseases. Whole blood anticoagulated with EDTA was used to assess MCV with an automated haematology analyser and serum was used to determine y-GT, ALT and AST activity. All blood measurements were conducted at the Institute of Laboratory Medicine, University Hospital Munich.

\section{Six-month follow-up measurements}

Higher levels of individual self-reported motivation and selfefficacy are presumed to be associated with better treatment outcomes in patients with $\mathrm{AD}[10,22,28]$. Moreover, study designs with a written informed consent process for study participation might be prone to selection bias since populations are not comparable with real-world settings [18]. Our approach to modify the classical procedure of written informed consent in a three-step procedure was approved a priori by the local ethics committee: (1) 5.5 months after the end of QWT we used the addresses available in the hospital database to inform all former inpatients who had received QWT for AD in written form about the study. (2) Trained interviewers then attempted to contact all former inpatients by telephone 6 months (+ maximum 10 days) after the end of their QWT. The protocol specified that 10 attempts to reach a patient could be made at different time points during the day, including weekends. Finally, (3) Successfully contacted patients were asked whether they agreed to participate in the study that they had been informed about (i.e. we obtained oral informed consent). Then, the trained interviewers immediately conducted the semi-structured self-report follow-up interview to assess all outcome variables, as follows: (1) continuous abstinence from the end of QWT until follow-up (i.e. no alcohol use [no single drink]; yes/no), (2) duration (in days) of continuous abstinence (i.e. reported achieved days of continuous abstinence with no single drink after the end of QWT; if patients reported continuous abstinence for the full 6 months, the duration of continuous abstinence was recorded as 180 days), (3) daily alcohol consumption based on 30-day TLFB [37]; mean daily alcohol consumption was calculated in grams: ethanol $[\mathrm{g}]=$ volume of beverages $\times 0.8 \mathrm{~g} / \mathrm{l} \times$ alcohol content [in $\%] / 100 \%$ ) and current abstinence (no alcohol consumption in 30-day TLFB). Finally, the interviewers assessed participant satisfaction with the inpatient QWT programme using the Munich Patient Satisfaction Scale (MPSS-24) [26] and investigated follow-up treatment after QWT. 


\section{Statistical analysis}

Statistical analysis was performed with IBM SPSS25.0 software (IBM, Armonk, NY, USA) with a significance level of $\alpha=0.05$. The Shapiro-Wilk test was used to assess whether data were normally distributed. Data were not transformed and we used both parametric (Student's $t$-test, Pearson's $r$ ) and nonparametric analyses (Mann-Whitney $U$ test, Kruskal-Wallis test, $\chi^{2}$ test, Fisher's exact test, Spearman's $\rho$ ), depending on the specific distribution properties. Correction for multiple testing using the family-wise error rate (FWE) was performed for families of tests within the same category (e.g. education, living situation). However, due to the exploratory nature of our analysis and our pre-defined aim of identifying candidate variables, no overall $P$-value correction was applied. Finally, baseline variables were used to predict both binary and continuous outcome features using logistic and linear regression analysis, respectively. Since our analyses on the relationship of biomarkers on relapse rates have to be considered exploratory rather than confirmatory, power analyses were not conducted.

\section{Results}

\section{Participant characteristics}

Of the 133 former inpatients included in the follow-up assessment (71.4\% men, $28.6 \%$ women; mean [SD] age: 47.4 [13.0] years), $n=59$ participants $(44.4 \%)$ reported being continuously abstinent since the end of their QWT. The mean (SD) duration of continuous abstinence was 111.44 (72.17) days. A total of $n=103$ participants (77.4\%) reported current abstinence. The mean (SD) daily alcohol consumption among all participants, calculated on the basis of TLFB, was 20.47 (57.40) g. The mean [SD] satisfaction score on the MPSS-24 was 22.40 [4.0] ( $n=132$ for satisfaction score; maximum score $=25$ ). The satisfaction score did not differ between participants who were currently abstinent and those who were currently drinking according to TLFB (22.28 [4.04] vs. 22.83 [3.86]; $U=2139.5 ; P=0.945$ ), nor did it differ between continuously abstinent participants and those who had relapsed (22.51 [3.82] vs. 22.32 [4.16]; $U=2139.5 ; P=0.945)$. Moreover, there was no significant correlation between the satisfaction score and daily alcohol consumption $(\rho=0.127 ; P=0.148)$ or days of continuous abstinence $(\rho=0.026 ; P=0.766)$. Table 1 presents all baseline subject characteristics and outcome results.

\section{Baseline sociodemographic and clinical variables associated with outcome variables}

Several sociodemographic and clinical variables differed significantly between abstinent and relapsed participants or correlated significantly with days of continuous abstinence or current daily alcohol consumption (Table 1). With regard to sociodemographic variables, a higher educational level was associated with both a higher probability for current abstinence $(P=0.010)$ and a lower alcohol consumption $(P=0.011)$. Furthermore, employment status was associated with longer continuous abstinence $(P=0.036)$. With regard to clinical variables, participants with current abstinence had received less oxazepam as withdrawal medication $(P=0.041)$. Higher oxazepam use during withdrawal treatment significantly correlated with higher daily alcohol consumption at the 6-month follow-up $(P=0.028)$. A higher number of previous detoxifications and alcohol treatments correlated with a lower number of days of continuous abstinence $(P=0.009)$. Moreover, the breath alcohol level at admission was lower in participants with current abstinence at the 6-month follow-up $(P=0.046)$. Higher breath alcohol levels at admission correlated with higher mean daily alcohol consumption at the 6-month follow-up $(P=0.022)$.

\section{Baseline blood biomarkers associated with outcome variables}

No single blood biomarker at admission significantly differed between abstinent and relapsed participants or correlated with any outcome variable. We did observe a significant correlation between the AST:ALT ratio and daily alcohol consumption at the 6-month follow-up $(r=0.232$; $P=0.007)$. A total of $n=59(44.4 \%)$ participants were high-risk patients, i.e. they fulfilled the above-mentioned risk threshold of an AST:ALT $>1.00$ and MCV $>90.0 \mathrm{fl}$ [17]. At the 6-month follow-up, $n=65 / 74$ (87.8\%) of the low-risk patients were currently abstinent compared with only $n=38 / 59(64.4 \%)$ of the high-risk patients $(P=0.001)$. Moreover, mean (SD) daily alcohol consumption was significantly higher among high-risk (42.25 [80.44] g) than in low-risk patients (3.10 [11.29], $p<0.001)$. Finally, high-risk patients achieved a shorter period of continuous abstinence (92.81 [71.26] days) than low-risk patients (126.28 [69.86] days, $P=0.027$ ) (Table 1).

\section{Ranking of baseline variables associated with outcome variables}

For each baseline variable, we determined whether it was significantly associated with each of the four outcome variables, i.e. continuous abstinence, duration (in days) of continuous abstinence, daily alcohol consumption based on TLFB and current abstinence. Table 2 highlights the ranking of the number of significant associations between the various baseline factors and the four outcome variables. The combination criterion of AST:ALT > 1.00 and MCV > $90.0 \mathrm{fl}$ at baseline was significantly associated with 3 of the 4 outcome variables; the baseline variables educational level, alcohol breath 
concentration and amount of used withdrawal medication were each significantly associated with 2 of the 4 outcome variables; and the AST:ALT ratio, number of previous inpatient treatments and employment status were significantly associated with 1 of the 4 outcome variables.

\section{Regression analysis}

To investigate effect sizes and to adjust for intervariable confounding effects, we performed regression analysis with those variables that were significantly associated with the respective outcome variable from Table 1 . We performed logistic regression to investigate the relationship between the identified baseline variables and current abstinence. Individuals who did not fulfill the combination criterion of AST:ALT > 1.00 and MCV > 90.0 fl at baseline had a 3.52 higher odds ratio to achieve abstinence 6 months after treatment than patients who fulfilled the criterion. Besides the combination criterion, also higher education provided significant higher odds ratios compared to basic school education (Table 3). By performing linear regression analysis for the continuous outcome variables, the fulfilled combination criterion significantly explained parts of the outcome variances of daily alcohol consumption (effect size $=0.347$ ) and days of continuous abstinence (effect size $=-0.190$ ). Besides that, only breath alcohol level at admission significantly predicted daily alcohol consumption 6 months later and number of previous inpatient treatments significantly explained parts of the variance of the achieved days of continuous abstinence (Table 4).

\section{Discussion}

The aim of our naturalistic longitudinal study in people who had received extended alcohol withdrawal treatment was to analyse the potential of individual and combined routine blood biomarkers and clinical and sociodemographic variables to predict outcome 6 months later. $48.8 \%$ of inpatients were successfully contacted and interviewed 6 months after withdrawal treatment, whereas $51.2 \%$ could not be reached. A significant difference between the two groups could only be detected for two baseline parameters (migration status and liver disease detected by ultrasound). All other baseline features including blood biomarkers did not significantly differ between the follow-up group and the non-follow-up group. Hence, by receiving informed consent not during withdrawal treatment but 6 months later during the followup survey, we were able to reduce potential selection bias compared to standard a priori written informed consent procedures [18]. Of note, a previous study in an outpatient setting also assessed AST, ALT, GGT and MCV with a 1-year follow-up period in a longitudinal naturalistic approach in patients with AD. This study used written informed consent before study participation and reported a subsequent dropout rate of $44 \%$ [14].

Table 2 Ranking of baseline variables dependent on the number of significant associations with outcome variables

\begin{tabular}{|c|c|c|c|c|c|}
\hline \multirow[t]{2}{*}{ Baseline factor } & \multirow{2}{*}{$\begin{array}{l}\text { No. of associations between base- } \\
\text { line factor and outcome variable }\end{array}$} & \multicolumn{4}{|l|}{ Outcome variable } \\
\hline & & Current abstinence* & $\begin{array}{l}\text { Continuous } \\
\text { abstinence* }\end{array}$ & $\begin{array}{l}\text { Daily alcohol } \\
\text { consumption }\end{array}$ & $\begin{array}{l}\text { Days of } \\
\text { continuous } \\
\text { abstinence }\end{array}$ \\
\hline AST:ALT $>1.00+\mathrm{MCV}>90.0 \mathrm{fl}$ & 3 & $P=0.001$ & $P=0.069$ & $P<0.001$ & $P=0.027$ \\
\hline Education level & 2 & $P=0.010$ & - & $P=0.011$ & - \\
\hline Breath alcohol level & & $P=0.046$ & $P=0.088$ & $P=0.022$ & $P=0.068$ \\
\hline Dose of oxazepam needed & & $P=0.041$ & - & $P=0.028$ & - \\
\hline AST:ALT ratio & 1 & $P=0.087$ & - & $P=0.007$ & - \\
\hline Previous inpatient treatment & & - & $P=0.058$ & - & $P=0.009$ \\
\hline Employment status & & - & $P=0.091$ & - & $P=0.036$ \\
\hline $\mathrm{MCV}$ & $0 * *$ & - & $P=0.067$ & - & $P=0.079$ \\
\hline Previous alcohol-related seizure & & $P=0.082$ & - & $P=0.068$ & - \\
\hline Psychiatric comorbidity & & - & - & - & $P=0.066$ \\
\hline Type of discharge & & - & - & $P=0.069$ & - \\
\hline
\end{tabular}

Significant $P$ values $(P<0.05)$ are written in bold. Non-significant $P$ values $<0.1$ are specified, but $P$ values $>0.1$ are indicated as "-".

AST aspartate aminotransferase, $A L T$ alanine aminotransferase, $M C V$ mean cell volume of erythrocytes

*Current abstinence: no alcohol consumption reported in 30-day timeline follow-back; continuous abstinence: no alcohol consumption since the end of inpatient withdrawal treatment

**Baseline variables with no significant associations and a $P$ value $\leq 0.1$ are included in the table, but baseline variables with no significant associations and a $P$ value $>0.1$ are not 
Table 3 Logistic regression of the relationship between identified baseline variables on current abstinence

\begin{tabular}{lrrrrrr}
\hline $\begin{array}{l}\text { Predictors for current abstinence (logistic } \\
\text { regression) }\end{array}$ & Wald & $d f$ & Sig & Exp(B) & \multicolumn{2}{l}{$\begin{array}{l}\text { 95\% C.I. for } \\
\text { EXP(B) }\end{array}$} \\
\cline { 4 - 7 } & & & & & Lower & Upper \\
\hline Breath alcohol level & 0.836 & 1 & 0.361 & 0.720 & 0.355 & 1.457 \\
Education & 11.021 & 3 & $\mathbf{0 . 0 1 2}$ & & & \\
Secondary school to end of year 10a & 3.901 & 1 & $\mathbf{0 . 0 4 8}$ & $\mathbf{3 . 8 9 2}$ & 1.010 & 14.992 \\
Secondary school to end of year 12/13 & 0.227 & 1 & 0.634 & 0.728 & 0.196 & 2.696 \\
University/college & 6.362 & 1 & $\mathbf{0 . 0 1 2}$ & $\mathbf{5 . 6 6 5}$ & 1.472 & 21.801 \\
AST:ALT $>1.00+$ MCV $>90.0 \mathrm{fl}^{\mathrm{b}}$ & 6.154 & 1 & $\mathbf{0 . 0 1 3}$ & $\mathbf{3 . 5 2 0}$ & 1.302 & 9.513 \\
Dose of oxazepam needed & 0.466 & 1 & 0.495 & 0.998 & 0.992 & 1.004 \\
\hline
\end{tabular}

Logistic regression model of the relationship between identified baseline and current abstinence. Independently significant associations $(P<0.05)$ are written in bold. Model statistics: $\chi^{2}=22.723, d f=6, P<0.001$, Nagelkerke's $R$ square $=0.262$

$D f$ degrees of freedom, Wald $\chi^{2}$ value, Sig. $=$ two-tailed $P$ value

aEducational level vs. "secondary school to end of year 9"

${ }^{\mathrm{b}}$ Not fulfilled vs. fulfilled

\begin{tabular}{lcrcc}
\hline & $\begin{array}{l}\text { Standardized } \\
\text { coefficients beta }\end{array}$ & & Sig \\
& & & & \\
\hline A) Predictors for daily alcohol consumption (linear regression) & & & $\mathbf{2 . 0 4 0}$ & $\mathbf{0 . 0 4 4}$ \\
Breath alcohol level & -0.160 & -1.894 & 0.061 \\
Education & -0.108 & -1.108 & 0.270 \\
AST:ALT ratio & 0.347 & $\mathbf{3 . 5 3 4}$ & $\mathbf{0 . 0 0 1}$ \\
AST:ALT $>1.00+$ MCV $>$ 90.0 fl-fullfilled & 0.138 & 1.461 & 0.147 \\
Dose of oxazepam needed & & & \\
(B) Predictors for days of continuous abstinence (linear regression) & & -0.190 & $-\mathbf{2 . 1 7 7}$ & $\mathbf{0 . 0 3 1}$ \\
AST:ALT $>1.00+$ MCV $>90.0$ fl-fullfilled & -0.219 & $-\mathbf{2 . 5 2 5}$ & $\mathbf{0 . 0 1 3}$ \\
Previous inpatient treatment & 0.048 & 0.548 & 0.584 \\
Employment status-Yes & & &
\end{tabular}

(A) Linear regression model of the relationship between identified baseline variables on daily alcohol consumption. Independently significant associations $(\mathrm{P}<0.05)$ are written in bold. Model statistics: $F=6.092$, $P<0.001, R$ square $=0.209$. (B) Linear regression of the relationship between identified baseline variables on days of continuous abstinence. Model statistics: $F=4.993, P=.003, R$ square $=0.107$
Table 4 Linear regression between identified baseline variables and daily alcohol consumption and continuous abstinence time than low-risk patients and had a significantly higher mean daily alcohol consumption (Table 1). Moreover, the combination criterion was significantly associated with more outcome variables ( 3 out of 4 ) than any other biological and non-biological factors (Table 2). Finally, we performed a regression analysis to estimate effect sizes or odd ratios and to adjust for confounding within the tested variables. Regression analysis highlighted that the combination criterion significantly predicted the three associated outcome variables. Moreover, all regression models that were based on the significant baseline variables from Table 1 provided significant models to predict part of the respective outcome variance.

In our study, $44.4 \%$ of the participants reported being continuously abstinent 6 months after inpatient treatment. This finding is consistent with previous studies reporting a continuous abstinence for a significantly shorter period of 
relapse rate of approximately $60 \%$ after alcohol treatment $[11,27]$. Also in line with previous studies was our finding that the number of previous detoxifications [39] and sociodemographic factors such as employment status and a higher level of education [38] were associated with better treatment outcomes. However, a direct comparison with other studies is not possible, due to several differences between studies (e.g. treatment setting, study design and follow-up period).

In the current study, individual blood biomarkers were not significantly associated with the outcome variables and only the baseline AST:ALT ratio significantly correlated with daily alcohol consumption at the 6-month follow-up. However, we identified the combined threshold criterion of an AST:ALT ratio $>1.00$ and MCV $>90.0 \mathrm{fl}$ as a potentially powerful tool to identify patients with a lower and higher risk for relapse after withdrawal treatment. We did not use the threshold criterion from Kawachi et al. [17] as a screening tool to identify patients with $\mathrm{AD}$, but as a potential predictor for future outcomes in patients with AD. It should be noted that at admission all participants met the criteria for AD according to ICD-10, but less than half of them met the threshold cut-offs of Kawachi et al. that were originally applied to identify AD patients. Remarkably, the combined threshold criterion was significantly associated with 3 out of 4 outcome variables and thus outperformed all other baseline variables (Table 2). High-risk patients reported current alcohol consumption significantly more often at the 6-month follow-up, were continuously abstinent for significantly shorter periods and consumed significantly more alcohol per day compared with low-risk patients. Only continuous abstinence as an outcome variable (which was lower in highrisk patients) did not reach the level of significance, which might be due to the low sample size (Table 1). Remarkably, also when we adjusted for potentially confounding effects of other candidate baseline variables by performing regression analysis, the combination threshold criterion of AST:ALT $>1.00$ and MCV $>90.0 \mathrm{fl}$ significantly predicted parts of the outcome variables.

To our knowledge, only a few studies have investigated in cohorts of patients with AUD whether baseline values of routine blood biomarkers are associated with AUD-relevant outcome variables. Maenhout et al. [23] suggested that ALT and GGT were predictors for drunk-driving recidivism. Florez and colleagues used all indirect alcohol blood biomarkers (GGT, AST, ALT, AST:ALT ratio and MCV) that were applied in this study and revealed the potential of indirect alcohol markers [14]. However, they had a more general interpretation of good clinical response without detailed distinction of abstinence or daily alcohol consumption. Moreover, there were two assessment time points: one at baseline before treatment and one after 6 months when the active intervention period was completed. Biomarkers at both time points were tested for their potential to predict the outcome after 18 months. For 18 months' prediction, 6 months biomarkers were more important than baseline parameters [14]. Moreover, GGT was previously identified as a potential predictor and was part of algorithms that applied GGT among other factors to calculate abstinence from drinking $[1,15,16]$. Our analyses confirmed that biological variables, already determined at admission, might help to predict long-term abstinence and the risk of relapse. However, in our study, only the combination of blood biomarkers was significantly associated with 6-months outcome variables.

A multitude of clinical decision limits can be applied to the De Ritis or AST:ALT ratio [7]. An AST:ALT ratio > 1.0 might be an indicator of liver fibrosis/cirrhosis or a resolving alcoholic hepatitis although $>1.0$ but $<1.5$ could be also a healthy condition in women, whereas an AST:ALT ratio $\geq 2$ in adults is associated with worse prognosis of relevant liver damage [7]. The MCV represents a measure for the average size of erythrocytes and is an established biomarker for alcohol abuse and AD [9]. Nevertheless, elevated MCV levels being above the established upper limit of $90 \mathrm{fl}$ might also be affected by other factors, such as e.g. smoking behaviour, poor nutrition and aging [33]. Despite its low sensitivity as a single biological screening marker to detect alcoholism, MCV can enhance sensitivity to detect alcoholism when combined with other alcohol-specific markers such as e.g. GGT [30]. A previous large US population-based study found that these markers identify very heavy alcohol drinking but fail to identify potentially harmful lower levels of alcohol intake [21].

Despite a range of uncertainty, indirect alcohol blood biomarkers and their combinations could reflect the degree of previous alcohol consumption that also reflects the severity of $\mathrm{AD}$ [35]. Moreover, it is very likely that the severity of $\mathrm{AD}$ is also linked to the risk of relapse after alcohol withdrawal treatment [6]. Therefore, blood biomarkers might help to identify patients with more severe $\mathrm{AD}$ and a higher risk of relapse.

This study has several limitations. We did not assess motivation or self-efficacy in participants, although both are associated with treatment outcome [10, 22, 28]. Moreover, the follow-up data are based on self-reports and did not include collateral reports [41] or biomarkers to validate self-reported drinking behaviour. However, previous studies revealed that self-reported alcohol consumption correlates with blood biomarkers [4, 34]. Furthermore, the sample size was relatively small and is based on only one centre with a limited follow-up period although 6 months' outcome is a good predictor for 5-year abstinence [40]. Therefore, our findings need to be confirmed in a multicentre study with a larger study population. In this study, we did not assess 
biomarkers like carbohydrate-deficient transferrin (CDT) or direct-ethanol metabolites such as phosphatidylethanol (PEth), ethyl glucuronide (EtG) or ethylsulfate (EtS) [42]. On the other hand, the use of those specific molecules also entails shortcomings that need to be considered. In contrast to AST, ALT, GGT and MCV that are standard parameters in clinical laboratories, these tests are more expensive and only available in specialized centres [3]. Moreover, the direct biomarkers might be more powerful to detect relapse and monitor abstinence but less powerful to monitor clinically relevant chronic cellular impairments in blood samples that could also reflect the somatic severity of AD.

\section{Conclusion}

In summary, this study highlights that, besides sociodemographic and clinical factors, routinely assessed indirect alcohol biomarkers might be useful in the future prediction of long-term outcome in alcohol withdrawal treatment and the identification of patients who have a more severe degree of AD and/or higher risk of relapse. Previously, Neumann and Spies [31] suggested using defined outcome variables to determine the value of single or combined blood biomarkers for future clinical decision-making algorithms with regard to treatment as usual or intensified treatment. Indirect alcohol biomarkers might support future treatment algorithms in daily routine care. Our study might help to pave the way for personalized medicine in AUD treatment with an evidencebased risk stratification and personalized risk prediction that comprises both biological and non-biological factors to identify high-risk patients who might need more intensified treatment and/or relapse prevention.

Acknowledgements Open Access funding provided by Projekt DEAL. The authors thank Jacquie Klesing, BMedSci (Hons), Board-certified Editor in the Life Sciences (ELS), for editing assistance with the manuscript. Ms. Klesing received compensation for her work from the LMU Munich, Germany. We also thank the administrative staff and addiction unit staff at the Department of Psychiatry and Psychotherapy, University Hospital, LMU Munich, Munich, Germany, for their support. Finally, we thank all patients for their participation.

Author contributions FR, EW, JW, SB, EH, GK conceptualized the present study. FR, EW, JW, SB conducted the present study. FR, EW, JW, DP conducted statistical analyses. FR, EW, JW, GB, KA, OP, EH, GK conducted the background literature review and contributed to the interpretation of the findings. FR, EW wrote the manuscript's first draft. All authors have contributed to editing subsequent drafts and have approved the final manuscript.

Funding This research was not supported by any specific grant from funding agencies in the public, commercial, or not-for-profit sectors. FR and DP were supported by the Else Kröner-Fresenius Foundation (Residency/PhD track of International Max Planck Research School for Translational Psychiatry).

\section{Compliance with ethical standards}

Conflict of interest statement The authors declare that they have no conflict of interest.

Open Access This article is licensed under a Creative Commons Attribution 4.0 International License, which permits use, sharing, adaptation, distribution and reproduction in any medium or format, as long as you give appropriate credit to the original author(s) and the source, provide a link to the Creative Commons licence, and indicate if changes were made. The images or other third party material in this article are included in the article's Creative Commons licence, unless indicated otherwise in a credit line to the material. If material is not included in the article's Creative Commons licence and your intended use is not permitted by statutory regulation or exceeds the permitted use, you will need to obtain permission directly from the copyright holder. To view a copy of this licence, visit http://creativecommons.org/licenses/by/4.0/.

\section{References}

1. Aguiar P, Neto D, Lambaz R, Chick J, Ferrinho P (2012) Prognostic factors during outpatient treatment for alcohol dependence: cohort study with 6 months of treatment follow-up. Alcohol Alcohol 47:702-710

2. Alatalo P, Koivisto H, Puukka K, Hietala J, Anttila P, Bloigu R, Niemela O (2009) Biomarkers of liver status in heavy drinkers, moderate drinkers and abstainers. Alcohol Alcohol 44:199-203

3. Andresen-Streichert H, Muller A, Glahn A, Skopp G, Sterneck M (2018) Alcohol biomarkers in clinical and forensic contexts. Dtsch Arztebl Int 115:309-315

4. Babor TF, Steinberg K, Anton R, Del Boca F (2000) Talk is cheap: measuring drinking outcomes in clinical trials. J Stud Alcohol 61:55-63

5. Batra A, Muller CA, Mann K, Heinz A (2016) Alcohol dependence and harmful use of alcohol. Dtsch Arztebl Int 113:301-310

6. Blaine SK, Sinha R (2017) Alcohol, stress, and glucocorticoids: from risk to dependence and relapse in alcohol use disorders. Neuropharmacology 122:136-147

7. Botros M, Sikaris KA (2013) The de ritis ratio: the test of time. Clin Biochem Rev 34:117-130

8. Charlet K, Rosenthal A, Lohoff FW, Heinz A, Beck A (2018) Imaging resilience and recovery in alcohol dependence. Addiction 113:1933-1950

9. Conigrave KM, Davies P, Haber P, Whitfield JB (2003) Traditional markers of excessive alcohol use. Addiction 98(Suppl 2):31-43

10. Dale V, Heather N, Adamson S, Coulton S, Copello A, Godfrey C, Hodgson R, Orford J, Raistrick D, Tober G, Team UR (2017) Predicting drinking outcomes: evidence from the united kingdom alcohol treatment trial (ukatt). Addict Behav 71:61-67

11. Dawson DA, Grant BF, Stinson FS, Chou PS, Huang B, Ruan WJ (2005) Recovery from dsm-iv alcohol dependence: United states, 2001-2002. Addiction 100:281-292

12. Degenhardt L, Charlson F, Ferrari A, Santomauro D, Erskine H, Mantilla-Herrara A, Whiteford H, Leung J, Naghavi M, Griswold M, Rehm J, Hall W, Sartorius B, Scott J, Vollset SE, Knudsen AK, Haro JM, Patton G, Kopec J, Carvalho Malta D, Topor-Madry R, McGrath J, Haagsma J, Allebeck P, Phillips M, Salomon J, Hay S, Foreman K, Lim S, Mokdad A, Smith M, Gakidou E, Murray C, Vos T (2018) The global burden of disease attributable to alcohol and drug use in 195 countries and territories, 1990-2016: a systematic analysis for the global burden of disease study 2016 . Lancet Psychiatr 5:987-1012 
13. Durazzo TC, Gazdzinski S, Yeh PH, Meyerhoff DJ (2008) Combined neuroimaging, neurocognitive and psychiatric factors to predict alcohol consumption following treatment for alcohol dependence. Alcohol Alcohol 43:683-691

14. Florez G, Saiz PA, Garcia-Portilla P, De Cos FJ, Dapia S, Alvarez S, Nogueiras L, Bobes J (2015) Predictors of posttreatment drinking outcomes in patients with alcohol dependence. Eur Addict Res 21:19-30

15. Gueorguieva R, Wu R, O'Connor PG, Weisner C, Fucito LM, Hoffmann S, Mann K, O'Malley SS (2014) Predictors of abstinence from heavy drinking during treatment in combine and external validation in predict. Alcohol Clin Exp Res 38:2647-2656

16. Hinton DJ, Vazquez MS, Geske JR, Hitschfeld MJ, Ho AMC, Karpyak VM, Biernacka JM, Choi DS (2017) Metabolomics biomarkers to predict acamprosate treatment response in alcoholdependent subjects. Sci Rep 7:2496

17. Kawachi I, Robinson GM, Stace NH (1990) A combination of raised serum ast: Alt ratio and erythrocyte mean cell volume level detects excessive alcohol consumption. N Z Med J 103:145-148

18. Kho ME, Duffett M, Willison DJ, Cook DJ, Brouwers MC (2009) Written informed consent and selection bias in observational studies using medical records: systematic review. BMJ 338:b866

19. Kokkevi A, Hartgers C (1995) Europasi: European adaptation of a multidimensional assessment instrument for drug and alcohol dependence. Eur Addict Res 1:208-210

20. Kravos M, Malesic I (2010) Glutamate dehydrogenase as a marker of alcohol dependence. Alcohol Alcohol 45:39-44

21. Liangpunsakul S, Qi R, Crabb DW, Witzmann F (2010) Relationship between alcohol drinking and aspartate aminotransferase: alanine aminotransferase (AST:ALT) ratio, mean corpuscular volume (MCV), gamma-glutamyl transpeptidase (GGT), and apolipoprotein A1 and B in the U.S. Popul J Stud Alcohol Drugs 71:249-252

22. Ludwig F, Tadayon-Manssuri E, Strik W, Moggi F (2013) Selfefficacy as a predictor of outcome after residential treatment programs for alcohol dependence: simply ask the patient one question! Alcohol Clin Exp Res 37:663-667

23. Maenhout TM, Poll A, Vermassen T, De Buyzere ML, Delanghe JR, Group RS (2014) Usefulness of indirect alcohol biomarkers for predicting recidivism of drunk-driving among previously convicted drunk-driving offenders: Results from the recidivism of alcohol-impaired driving (road) study. Addiction 109:71-78

24. Mann K, Hermann D (2010) Individualised treatment in alcoholdependent patients. Eur Arch Psychiatry Clin Neurosci 260(Suppl 2):S116-120

25. McDonald H, Borinskya S, Kiryanov N, Gil A, Helander A, Leon DA (2013) Comparative performance of biomarkers of alcohol consumption in a population sample of working-aged men in russia: the izhevsk family study. Addiction 108:1579-1589

26. Möller-Leimkühler AM, Dunkel R, Müller P, Pukies G, de Fazio $S$, Lehmann E (2002) Is patient satisfaction a unidimensional construct? Eur Arch Psychiatry Clin Neurosci 252:19-23

27. Monahan SC, Finney JW (1996) Explaining abstinence rates following treatment for alcohol abuse: a quantitative synthesis of patient, research design and treatment effects. Addiction 91:787-806
28. Moos RH, Moos BS (2006) Rates and predictors of relapse after natural and treated remission from alcohol use disorders. Addiction 101:212-222

29. Morley KC, Luquin N, Baillie A, Fraser I, Trent RJ, Dore G, Phung N, Haber PS (2018) Moderation of baclofen response by a gabab receptor polymorphism: Results from the bacald randomized controlled trial. Addiction 113:2205-2213

30. Mundle G, Ackermann K, Munkes J, Steinle D, Mann K (1999) Influence of age, alcohol consumption and abstinence on the sensitivity of carbohydrate-deficient transferrin, gamma-glutamyltransferase and mean corpuscular volume. Alcohol Alcohol 34:760-766

31. Neumann T, Spies C (2003) Use of biomarkers for alcohol use disorders in clinical practice. Addiction 98:81-91

32. Noone M, Dua J, Markham R (1999) Stress, cognitive factors, and coping resources as predictors of relapse in alcoholics. Addict Behav 24:687-693

33. Pavanello S, Snenghi R, Nalesso A, Sartore D, Ferrara SD, Montisci M (2012) Alcohol drinking, mean corpuscular volume of erythrocytes, and alcohol metabolic genotypes in drunk drivers. Alcohol 46:61-68

34. Polich JM (1982) The validity of self-reports in alcoholism research. Addict Behav 7:123-132

35. Rosoff DB, Charlet K, Jung J, Lee J, Muench C, Luo A, Longley M, Mauro KL, Lohoff FW (2019) Association of high-intensity binge drinking with lipid and liver function enzyme levels. JAMA Netw Open 2:e195844

36. Saxena S, Thornicroft G, Knapp M, Whiteford H (2007) Resources for mental health: Scarcity, inequity, and inefficiency. Lancet 370:878-889

37. Sobell LC, Sobell MB (1992) Timeline follow-back: a technique for assessing self-reported alcohol consumption. Measuring alcohol consumption: psychosocial and biochemical methods. Humana Press, Totowa, pp 41-72

38. Sofin Y, Danker-Hopfe H, Gooren T, Neu P (2017) Predicting inpatient detoxification outcome of alcohol and drug dependent patients: the influence of sociodemographic environment, motivation, impulsivity, and medical comorbidities. J Addict 2017:6415831

39. Soyka M, Schmidt P (2009) Outpatient alcoholism treatment24-month outcome and predictors of outcome. Subst Abuse Treat Prevent Policy 4:15

40. Weisner C, Ray GT, Mertens JR, Satre DD, Moore C (2003) Short-term alcohol and drug treatment outcomes predict longterm outcome. Drug Alcohol Depend 71:281-294

41. Whitford JL, Widner SC, Mellick D, Elkins RL (2009) Self-report of drinking compared to objective markers of alcohol consumption. Am J Drug Alcohol Abuse 35:55-58

42. Wurst FM, Thon N, Yegles M, Schruck A, Preuss UW, Weinmann W (2015) Ethanol metabolites: their role in the assessment of alcohol intake. Alcohol Clin Exp Res 39:2060-2072 